302 Poggiale, über einiye Verbindungen d. Cyanquecksilbers.

$\mathrm{PtCy}, \mathrm{ZnCy}, \mathrm{NH}_{3}$, HO.

Nickelsalz ( $\mathrm{PtCy}, \mathrm{NiCy}, \mathrm{NH}_{3}, \mathrm{HO}$ ) erhält man durch Fällen des Gınelin'schen Salzes mit einer zur Ausfällung nicht hinreichenden Menge von in Ammoniak gelöstem Nickeloxydulhydrat. Violetle, nadelförmige Krystalle.

Kobaltsalz, als fleischfarbiges Pulver durch Fällen von Kaliumplatincyanür mit einer Lösung von Chlorkobalt in einem Gemenge von Ammoniak und kohlensaurem Ammoniak. Beim Erhitzen wird es blau, verliert schon bei $120^{\circ}$ wenig Ammoniak.

\title{
Ueber einige neue Verbindungen des Cyanquecksilbers;
} von Poggiale.

Das Cyanquecksilber besitzt, sowie das Quecksilberchlorid, elektronegative Eigenschaften und verbindet sich leicht mit Bromiden, Jodiden, Chloriden und sellsst mit Sauerstoffsalzen, von denen jedoch bis jelzt nur noch wenige beschrieben worden sind. Man erbält diese Verbindungen meist, wenn man Cyanquecksilber zu einer kalt gesältigten Lösung, z. B. eines Chlorids, setzt, dann filtrirt und die Flüssigkeit bei sehr' gelinder Wärme verdampft. Bisweilen mufs man auch, um schöne Krystalle zu erhallen, im Vacurm verdunsten. Bei der Analyse wurde die mil Salzsäure angesäuerte und elwas erwärmte Flüssigkeit anhaltend mit Schwefelwasserstoffgas behandelt. Die Verbindung von Schwefelquecksilber mit Cyanquecksilber, welche anfänglich enistelit, verwandelt sich bei längerer Einwirkung des Schwefelwasserstoffs in reines Schwefelguecksilbers. Aus der vom Schwefelquecksilber befreiten Flüssigkeit konnte das andere Salz meist Jurch blofses Verdampfen und Glühen erhalten werden. Der Wassergehalt der Salze wurde theils dadurch bestimmt, dafs man dieselben auf dem Sandbade gelinde erwärmte, theils dadurch, 
Poggiale, über einige Verbindungen d. Cyanquecksilbers. 303

dafs nan sie über concentrirler Schwefelsäure oder auch im Vacuun trocknete.

Cyanquecksilber mit Salmiak. Durch Auflösen beider Substanzen und gelindes Eindampfen erhalten, krystallisirt es in langen Nadeln, die sich leichter in lieifsem als in kaltem Wasser lösen und an der Luft undurchsichtig werden. Von der Hitze wird das Salz zersetzt und entwickelt Kohlensäure, Cyan, Quecksilber - und Salmiakdämpfe. Salzsäure und Schwefelsäure entwickeln daraus Blausäure, bei erhöhter 'Temperatur letzteres Cyan, schweflige Säure, Kohlensäure und Salzsäure. Die Formel des Salzes ist : $2 \mathrm{Hg} \mathrm{Cy}+\mathrm{NH}_{3}, \mathrm{H} \mathrm{Cl} *$ ]). Ueberlä[st man die Mutterlauge des erwähnten Salzes einer langsamen Verdunstung, so erhält man glänzende, dreiseilige Blättchen eines Salzes, dessen Formel $\mathrm{Hg} \mathrm{Cy}+2 \mathrm{NH}_{3}, \mathrm{H} \mathrm{Cl}$ ist.

Cyanquecksilber mit Chlornatrium. Dieses krystallisirt in schönen durchsichtigen Nadeln, welche kein Krystallwasser enthalten. Das Salz löst sich leicht in Wasser, besonders in kochendem, aber wenig in Alkohol. Es hat die Formel : $\mathrm{Hg} \mathrm{Cy}, \mathrm{NaCl}$.

Cyanquecksilber mit Chlorbarium. Dieses Salz bildet schöne vierseilige, durchscheinende Prismen, welche am Ende schief abgeschnitten sind und an der Luft verwillern, ohne jedoch zu zerfallen. In der Wärme geben sie selır leicht ihr Krystallisationswasser $a b$, in stärkerer Hilze werden sie zersetzt. Durch schwefelsaure Salze wird ein Niederschlag hervorgebracht, ebenso durch lösliche kohlensaure Salze und durch Schwefelwasserstoffgas. Die Formel ist : $\mathrm{Hg} \mathrm{Cy}+\mathrm{Ba}^{* *}$ ) $\mathrm{Cl}+4 \mathrm{HO}$.

Cyanquecksilber mit Chlorstrontium. Durch freiwillige Verdunslung eines Gemisches von 2 Aeq. Cyanquecksilber u. 1 Aeq. Chlorstrontium schieden sich feine seidenglänzende, zu Gruppen vereinigte Prismen aus, welche sich sehr leicht in Wasser lösen

$\left.{ }^{*}\right) \mathrm{N}=175,08, \mathrm{Cl}=450,00, \mathrm{Hg}=1250,90$.

**) $\mathrm{Ba}=858,03$. 
304 Poggiale, über einige Verbindungen d. Cyanguecksilbers.

und an der Luft schon nach einigen Stunden ihren Glanz verlieren. Das Salz wird, so wie das vorhergehende, durch schwefelsaure und kohlensaure Salze, so wie durch Schwefelwasserstufl zersetzl. Seine Formel ist: $\left.2 \mathrm{Hg} \mathrm{Cy}+\mathrm{Sr}^{*}\right) \mathrm{Cl}+6 \mathrm{HO}$.

Cyanquecksilber mit Chlorcalcium. Wenn man zu 2 Aeq. Cyanquecksilber 1 Aeq. Chlorcalcium zusetzt, so bildet sich bej gehörig anhaltender Wärme ein in kleinen prismatischen Nadeln krystallisirendes Salz, welches sich leicht in Wasser; löst und an der Luft verwiltert. Seine Formel ist : $2 \mathrm{Hg} \mathrm{Cy}+\mathrm{CaCl}+6 \mathrm{HO}$.

Cyanquecksilber mit Chlormagnesium. Dieses Salz krystallisirt in prismatischen Nadeln, hält sich an trockner Luft und zerfliefst bei Gegenwart von etwas Feuchtigkeit. Es enthält $2 \mathrm{HgCy}, \mathrm{Hg} \mathrm{Cl}+2 \mathrm{HO}$.

Cyanquecksilber mit Hanganchloriir. Eine Lösung von 1 Aeq. Chlormangan und 1 Aeq. ${ }^{\circ}$ Cyanquecksilber giebt durch freiwillige Verdunslung durchscheinende, farblose, vierseitige Tafeln. Dieselben sind leicht löslich in Wasser und verwittern an der Luft. Die Formel derselben ist : $\mathrm{Hg} \mathrm{Cy}, \mathrm{Mn} \mathrm{Cl}+3 \mathrm{HO}$.

Cyanquecksilber mit Chlorzink giebt gerade vierseitige Säulen, welche sich in Wasser lösen und an der Luft verwiltern. Sie bestehen aus $2 \mathrm{Hg} \mathrm{Cy}, \mathrm{ZnCl}+6 \mathrm{HO}$.

Cyanquecksilber mit Chlornickel krystallisirt schwer, ist blaugrün, zerfliefslich und sehr löslich in Wasser. Walırscheinlich hat es die Formel: $\mathrm{Hg} \mathrm{Cy}, \mathrm{NiCl}+6 \mathrm{HO}$.

Cyanguecksilber und Chlorhobalt. Dieses Salz, welches ebenso wie die vorhergehenden dargestellt wird, krystallisirt in warzenförmigen, gelblich-röthlichen Gruppen, welche an der Luft röthlich werden und leicht verwittern. Die concentrirte Lösung des Salzes ist, wie heim Chlorkobalt, blau, die verdünnte rotl. Die Formel ist : $\mathrm{Hg} \mathrm{Cy}, 2 \mathrm{CoCl}+4 \mathrm{HO}$.

*) $\mathrm{Sr}=548,02$. 
Cyanquecksilber mit Chlorquecksilber. Utn diose Verbindung zu erhalten, löst man 1 Aeq. Cyanid und 1 Aeq. Chlorid in Wasser und verdanpft die filtrinte Flüssigkeit bei gelinder W ärme. Beim Abkühlen erhält man halbdurchscheinende, vierseilige Pyramiden, welche sich nicht an der Luft verändern und sich in Wasser lösen. Die Verbindung enthält : $\mathrm{HgCy}, \mathrm{HgCl}$.

Zinnchlorid scheint sich mit dem Cyanquechsilber zu verbinden, doch konnten licine deutlichen Kiystalle davon erhalten werden. Zinnchlorür dagegen zersetzt das Cyanquecksilber sehr schnell und giebt einen grauen Niederschlag von Quecksilber und Zinnoxyd, während sich ziemlich lebhaft Blausäure entwickelt.

Cyanquecksilber und Jodcalciom krystallisirt in schönen seidenglänzenden Nadeln, ist sehr leicht löslich und verwitlert an der Luft. Es hat die Formel: $2 \mathrm{HgCy}, \mathrm{Ca} J+6 \mathrm{HO}$.

Cyanquecksilber und ameisensaures Ammoniak krystallisirl in dreiseitigen Prismen. Bei $200^{\circ}$ zersetzt es sich in Wasser, Cyanwasserstoffsäure und Cyanquecksilber; unter dem Finflusse der Schwefelsäure in der Wärme entwickelt es Kohlenoxydgas. Es besteht aus $\mathrm{Hg} \mathrm{Cy}, \mathrm{C}_{4} \mathrm{H}_{2} \mathrm{O}_{3}, \mathrm{NH}_{3}$.

Cyanquecksilber mit chlorsaurem Kali. Diese Verbindung ist bereits von Gaillot und Poitevin dargestellt, aber noch nicht analysirt werden. Es krystallisirt in blättrigen Nadeln, ist löslich in Wasser, an der Luft unveränderlich und entzündet sich bei höherer Temperatur. Nach dem Verf. ist die Formel derselben : $2 \mathrm{HgCy}, \mathrm{KO}, \mathrm{CrO}_{3}$. (Compl. rend. XXIII. p. 752-766.)

\section{Einwirkung von Chlor auf Cyanquecksilber.}

Bou is *) selzte eine kochend gesältigte Lösung von Cyanquecksilber in directen Sonnenlicht der Einwirkung des Chlors

*) Aun. de Chim, et de Phys. T. XX. p. 446.

Anual. d. Chemic u. Phatm. I.Xiv. Ba. 3. Heft. 